\title{
Throughput Analysis of a Cooperative ARQ Scheme in the Presence of Hidden and Exposed Terminals
}

\author{
Jesus Alonso-Zarate • Luis Alonso • \\ Georgios Kormentzas • Rahim Tafazolli • \\ Christos Verikoukis
}

Published online: 23 July 2011

(C) Springer Science+Business Media, LLC 2011

\begin{abstract}
Cooperative Automatic Retransmission Request (C-ARQ) schemes exploit the broadcast nature of the radio channel by allowing those users which overhear a transmission to act as spontaneous relays when a packet has been received with errors at destination. Transmission takes place in two phases. First, the source transmits to the destination. In the case of error, retransmissions are then executed by the relays, providing the system with cooperative diversity. In this
\end{abstract}

This work has been supported by the research projects

PASSENGER (PIEF-GA-2008-219561), CENTENO

(TEC2008-06817-C02-02), and CO2GREEN

(TEC2010-20823).

J. Alonso-Zarate $(\varangle) \cdot$ Ch. Verikoukis

Centre Tecnològic de Telecomunicacions

de Catalunya (CTTC), Av. Carl Friedrich Gauss,

7, CTTC, 08860 Castelldefels, Barcelona, Spain

e-mail: jesus.alonso@cttc.es

Ch. Verikoukis

e-mail: cveri@cttc.es

\section{Alonso}

Department of Signal Theory and Comms.,

Universitat Politècnica de Catalunya (UPC),

Av.Esteve Terradas, 7, EETAC, 08860 Castelldefels,

Barcelona, Spain

e-mail: luisg@tsc.upc.edu

\section{G. Kormentzas}

Department of Information and Communication

Systems Engineering, University of the Aegean,

Karlovasi, Samos 83200, Greece

e-mail: gkorm@aegean.gr

\section{R. Tafazolli}

Centre for Communication Systems Research,

University of Surrey, Guildford, UK

e-mail: r.tafazolli@surrey.ac.uk paper we analyze how the addition of these relays to the communication modifies the well-known hidden and exposed terminal problems associated to any medium access control protocol based on carrier sensing. This work is focused on Carrier Sensing Multiple Access (CSMA) protocols, such as the one defined in the IEEE 802.11 Standard. The theoretical study presented in this paper has been supported by computer-based simulations.

Keywords PRCSMA • 802.11 • MAC . hidden terminals • exposed terminals • cooperative ARQ $\cdot \mathrm{C}-\mathrm{ARQ}$

\section{Introduction}

In this paper we analyze the hidden and exposed terminal problems in the context of Cooperative Automatic Retransmission Request (C-ARQ) schemes. When a C-ARQ scheme is executed at the Medium Access Control (MAC) layer, communication takes place in four steps whenever a data packet is received with unrecoverable errors at destination. First, the source transmits a data packet to the destination. Note that, due to the broadcast nature of the wireless channel, this transmission can be overheard by some of the stations within the transmission range of the transmitter besides the intended destination. Second, the destination broadcasts a Call for Cooperation (CFC) packet. This packet invites all the potential helpers, i.e., those users which were able to decode the original transmission from the source, to assist in the transmission. Some of them become active relays (helpers) and a cooperation phase is initiated. In the third step, all the active 
relays attempt to assist the destination by retransmitting copies of the original transmission. These copies might be exact, recoded, compressed, or simply amplified versions of the original transmission [1]. Although the relays or helpers might transmit orthogonally in time, frequency, or code, we will focus on timeorthogonal retransmissions, which might have a simpler implementation. Finally, the destination attempts to combine the different independent copies of the original packet and acknowledges, either positively or negatively, the reception or reconstruction of the original packet. The cooperation phase is finished.

Several works in the literature have evaluated C-ARQ schemes from a fundamental point of view [24]. These works focus on the analysis at the PHY layer, adopting an information theoretical approach, and usually assume simplified topologies with just one relay or in single-hop scenarios. These works have shown that C-ARQ can significantly increase the performance of wireless communications in terms of throughput, delay, energy consumption or even improved coverage. However, still more work has to be done in upper layers of the protocol stack in the light of practical application of C-ARQ. This is the motivation for the main contributions presented in this paper, which are:

1. Theoretical analysis of the channel utilization factor of a network based on Carrier Sensing Multiple Access (CSMA) in the presence of hidden and exposed terminals when a $\mathrm{C}$-ARQ scheme is executed at the MAC layer. Note that commercial standards such as the IEEE 802.11 or the IEEE 802.15.4 are based on CSMA, and thus the analysis presented in this paper applies for these standards.

2. Evaluation of a practical case study wherein the relays of a C-ARQ scheme transmit orthogonally in time.

Extensive computer simulations have been carried out to assess the accuracy of the theoretical analysis and, for the practical implementation of the C-ARQ, we have focused our work on the Persistent Relay CSMA (PRCSMA) protocol first described in [5] and further analyzed in [6]. PRCSMA is a MAC protocol based on the IEEE 802.11.

Very few works can be found in this topic so far. In [7], authors qualitatively discuss how the use of a single intermediate relay affects the channel reuse of the channel for their specific rDCF protocol. Korakis et al. [8] also discuss the effects of the use of a single, faster, relay in terms of channel reuse within the context of the CoopMAC protocol. They argue that since the overall transmission time of a packet can be reduced with the use of the relay, the effects of both hidden and exposed terminal problems are alleviated. However, up to our knowledge, there is no comprehensive theoretical analysis yet available on how the hidden and exposed terminal problems are handled by a C-ARQ when more than one relay can help in any generic CSMA-based network.

The rest of the paper is organized as follows. In Section 2 we explain the hidden and exposed terminal problems and we discuss them in the context of C-ARQ schemes. In Section 3 we analyze the utilization factor of a network executing a C-ARQ scheme at the MAC layer and taking into account both the hidden and the exposed terminal problems. In Section 4 we conduct both numerical evaluation and computer simulations to assess the performance of a network in the presence of hidden and exposed terminals when a C-ARQ scheme is executed at the MAC layer. Finally, Section 5 concludes the paper.

\section{Problem statement and discussion}

The presence of hidden and exposed terminals in CSMA-based wireless networks has a direct impact on the performance of the communications. We briefly describe these two problems as follows.

A hidden terminal lies in the transmission range of a receiver but it is out of the range of the transmitting station. Therefore, the hidden terminal is oblivious of the ongoing transmission and can initiate a new transmission that will cause a collision at the receiver. The occurrence of these collisions reduces the overall performance of the network. The duration of the ongoing transmission is referred to as the vulnerability period, as this is the period of time exposed to the possible interference of a hidden terminal.

An exposed terminal lies in the transmission range of a transmitting user but it is out of the transmission range of the receiver. Although this station could transmit without incurring in a collision at the received, it remains silent due to the busy channel detection. This effect reduces the overall channel utilization by stopping some stations from transmitting despite the fact that they would not cause a collision.

An example of hidden and exposed terminals is depicted in Fig. 1. In this figure, a node TX is transmitting a data packet to node RX. Node $\mathrm{H}$ is hidden to the transmission of TX. H senses the channel idle (is not in the transmission range of TX). Therefore, if $\mathrm{H}$ starts a transmissions, it will cause a collision at node RX. In its turn, node $\mathrm{E}$ is exposed to transmission of TX. Despite the fact that $\mathrm{E}$ could initiate a transmission to node A without incurring in a collision at node 
Fig. 1 Description of hidden and exposed terminal problems

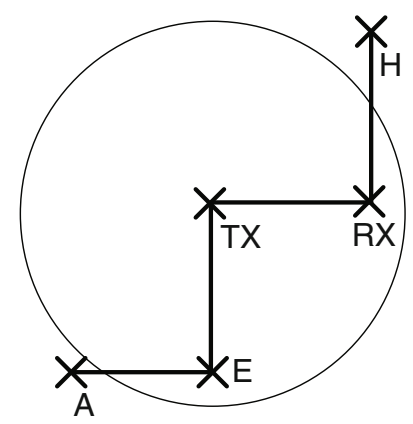

$\mathrm{RX}$, it cannot transmit due to the sensed activity from node TX.

We evaluate in this paper how the execution of a C-ARQ scheme at the MAC layer modifies these two problems. The fact that the active relays become transmitters and receivers at some point in time forces us to revisit the hidden and exposed terminal effects on the overall performance of the network.

The first observation is that the vulnerability period of a transmission is extended if retransmissions are required. Therefore, if a cooperation phase is initiated, then the channel has to be reserved for enough time as to ensure that the cooperation phase can be completed. For this time, collisions can occur due to the hidden terminal problem and the exposed terminal problem is exacerbated. However, the essence of the hidden terminal problem remains unaltered since a hidden terminal lies in the transmission range of the destination, and the destination does not change in the context of a C-ARQ scheme. In addition, it is worth observing that the relays can be hidden terminals for other third transmissions thus affecting the overall performance of the network. The increase of the offered load including retransmissions must be taken into account when evaluating the effects of the hidden terminals.

Regarding the exposed terminal problem, it has to be noted that whenever a destination station calls for cooperation, new transmitters appear in its surrounding area, changing the otherwise simple scenario formed by a transmitter and a receiver. The fact that some neighbors become active transmitters, and thus occupy the channel, extends the area exposed to the original transmission from the source to the destination.

In the next section we provide a comprehensive insight and analysis of the problem and we compute the throughput of a network considering the hidden and exposed terminal problems within the context of a C-ARQ scheme. We first consider the operation of the network without C-ARQ and we then discuss how the execution of a C-ARQ scheme with time-orthogonal relays modifies the analysis.

\section{Throughput analysis}

\subsection{Scenario and definitions}

We consider an ad hoc network formed by an arbitrary number of mobile stations spread out uniformly in a given network area. All the stations contribute equally to the total offered traffic load, which we assume to be generally distributed and to have a mean value of $g$ packets per second where $g$ includes originally generated packets and retransmissions (including those performed by the relays). The size of the data packets is also generally distributed and has a mean value of $P$ bits per packet. The network operates in finite load conditions. Therefore, we can define the throughput of the network as

$U(g)=U_{\mathrm{LOS}}(g) e_{2}(g)$,

where $U_{\text {LOS }}$ is the throughput of a network wherein all the stations are in the transmission range of each other (Line Of Sight, LOS) and $e_{2}$ is the throughput reduction factor due to the collisions caused by hidden terminals. For convenience and in order to clarify the notation, we drop henceforth the dependence of these terms with $g$ in the notation. The term $U_{\mathrm{LOS}}$ can be computed as

$U_{\mathrm{LOS}}=\frac{S}{B+I}$.

$S$ is the average effective data packet transmission time, $B$ is the average duration of a busy period (including the transmission of data and control as well as collisions), and $I$ is the average duration of an idle period wherein the channel remains idle.

\subsection{Ideal throughput of a CSMA-based network}

We focus on a network executing a CSMA-based MAC protocol similar to that of an IEEE 802.11 network. We consider a discrete time reference where events occur at the end of each slot, and we define $\tau$ as the duration of the time slot, following the definition of the standard of the IEEE 802.11 Standard [9]. We define $p$ as the probability that no packet arrives in a given time slot. Consequently, packet arrivals can be modeled as a Bernoulli process with probability of success $(1-p)$. Therefore, the number of consecutive idle slots until a packet arrival occurs follows a geometric distribution $(1-p) p^{(n-1)}$, where $n$ is the number of consecutive idle slots. Accordingly, the expected duration of an idle period can be expressed as

$I=\tau \sum_{n=1}^{\infty} n p_{n}=\tau \sum_{n=1}^{\infty} n(1-p) p^{n-1}=\frac{\tau}{1-p .}$ 
For the computation of the average busy period $(B)$, we define $p_{s}$ as the conditioned probability that a successful transmission occurs in a given slot, given that at least one arrival occurs in that slot. Let define $p_{1}$ as the probability that a single arrival occurs in a slot. Since the probability that at least one arrival occurs in a slot is given by $(1-p)$, the probability that a packet is successfully transmitted is given by

$p_{s}=\frac{p_{1}}{1-p}$.

Subsequently, the average number of consecutive slots for which at least one packet arrives is denoted by $n_{B}$ and computed as

$n_{B}=\sum_{n=1}^{\infty} n p(1-p)^{n-1}=\frac{1}{p}$.

According to these definitions, $n_{B} p_{s}$ is the average number of successful slots within a busy period, and $n_{B}\left(1-p_{s}\right)$ is the average number of collided slots within a busy period. Therefore, the expected duration of a busy period is given by

$B=T_{s} n_{B} p_{s}+T_{c} n_{B}\left(1-p_{s}\right)=\frac{T_{c}+p_{s}\left(T_{s}-T_{c}\right)}{p}$,

with $T_{s}$ and $T_{c}$ the duration of a successful and collided slots, respectively. The values for these two parameters are given in [10].

Lastly, we have to compute the value of $S$. To do so, we assume that data packets of length $P$ bits are transmitted at a certain rate $R$. The transmission time of these packets is $T_{p}=P / R$, and the average time for which payload information is transmitted in the channel is given by

$S=T_{p} n_{B} p_{s}=\frac{P p_{1}}{R p(1-p)}$.

Using Eqs. 3, 6, and 7 into Eq. 2, a closed-form expression for the average throughput can be obtained in LOS conditions.

\subsection{Throughput with hidden terminals}

Following the terms in Eq. 1, we now consider the hidden terminal problem to compute the value of $e_{2}$. In this case, the probability of success of a transmission depends on the probability that no node within the transmission range of the intended receiver initiates a transmission. This probability is derived in [11] within the context of a CSMA-based protocol and can be expressed as

$e_{2}=\left[\frac{I}{B+I} p^{T_{s} / \tau}\right]^{N-1}$,

where $N$ is the quotient of stations that are in LOS with a specific station to the stations that can affect the transmission of this station. The stations that can affect the current transmission are those that are at a maximum distance $r$ from the receiver and farther than $r$ from the transmitter and can thus be within the transmission range of the receiver but not the transmitter. Therefore, if $\rho$ is the nodes' density and $r$ is the transmission range of each station, then

$N=\rho \pi(2 r)^{2} / \rho \pi r^{2}=4$.

\subsection{Throughput with exposed terminals}

We now turn the focus to the analysis of the exposed terminal problem. It is important to note that this problem does not reduce the throughput as computed with Eq. 1, but it prevents it from becoming higher. In fact, it is possible to express the ideal throughput that would be achievable in the case that exposed stations could know when to transmit and when to defer the transmissions to avoid either misused or waste of resources as

$U=U(g)+U\left(g_{e}\right)$.

In this expression, $g_{e}$ represents the additional data traffic rate (packets per second) that would be transmitted in the network if the exposed terminal problem was solved. In order to analyze this parameter, let now take a look at Fig. 2. $\mathrm{T}$ is transmitting at a certain

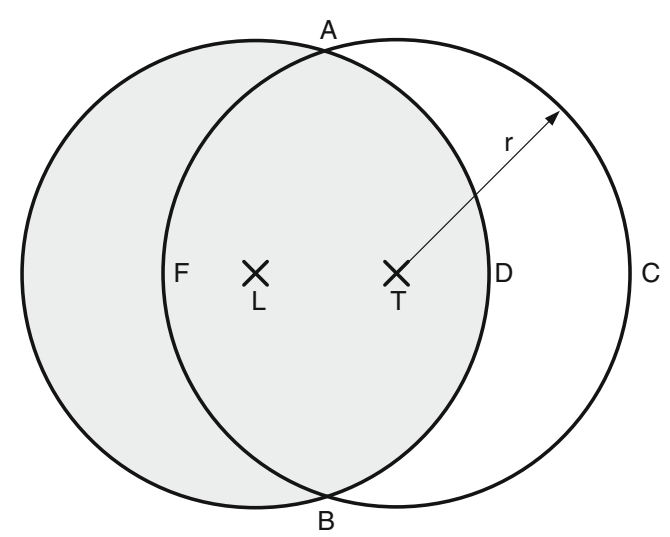

Fig. 2 Analysis of the exposed terminal problem 
moment, and its transmission range is represented in the figure by a circle of radius $r$ centered at $\mathrm{T}$. We denote by $E$ the area of that circle. If the transmission from $\mathrm{T}$ is referred to a station within the region $E 1$, delimited by the points ABFD in the figure, then $\mathrm{L}$ should remain silent to avoid a collision even in the case it wants to transmit a packet. On the other hand, if the transmission of $\mathrm{T}$ is referred to a station within the region $E 2$, limited by the points $\mathrm{ABDC}$ in the figure, then $\mathrm{L}$ is exposed and it will unnecessarily defer its transmission as it would not collide with the ongoing transmission from T. However, following the rules of the CSMA-based MAC protocol of the IEEE 802.11, $\mathrm{L}$ will remain silent. Therefore, assuming that the stations are uniformly distributed throughout the network area and that transmissions occur equiprobably to all stations, then there is a proportion $E 2 / E$ of deferral periods performed by station $\mathrm{L}$ that are not necessary. Considering the regular operation of the IEEE 802.11, the fraction $E 2 / E$ can be found in [12] equal to 0.42 .

Let us assume that there exists an ideal mechanism that allows stations to know when they should transmit or not, always respecting ongoing transmissions but avoiding the exposed terminal problem. The probability that a deferral period occurs because the medium is busy is determined by the probability that the medium is busy, which is equal to $B /(B+I)$, times the probability that a packet arrives within a slot, which is $(1-p)$. Therefore, if stations can know when to transmit during another transmission and when not, then a ratio

$\beta=\left(\frac{B}{B+I}\right)(1-p)\left(\frac{E_{2}}{E}\right)$

of additional transmissions will occur in the medium simultaneously with ongoing transmissions and without incurring in a collision. This means that an extra proportion of traffic load $g_{e}$, also expressed in packets per second, would be transmitted in the network, such that the probability that a packet arrives in a time slot is $\beta$. Then, the computation of $g_{e}$ from $\beta$ is simple if we know the distribution describing the packet generation rate. An example of this will be presented later in Section 4 where some numerical evaluation is performed.

\subsection{Throughput analysis with C-ARQ}

Let now investigate how the use of a C-ARQ scheme modifies the analysis described throughout the previous subsections. It is worth observing that the duration of a successful transmission can be expressed as

$T_{s}^{\prime}=T_{s}\left(1-p_{e}\right)+\left(T_{s}+T_{\mathrm{COOP}}\right) p_{e}$, where $p_{e}$ is the probability that a packet is received with errors and thus cooperation is requested. Therefore, if there is no error, the duration of a transmission is determined by the regular operation of the IEEE 802.11 Standard, denoted by $T_{s}$. However, in the case of an error, the duration of a successful transmission is equal to $T_{s}$ plus the duration of the cooperation phase, denoted by $T_{\mathrm{COOP}}$. This duration is determined by the number of required retransmissions and the MAC protocol used to coordinate the relays. Accordingly, it will be necessary to consider this new transmission time when either evaluating the hidden or the exposed terminal problems.

First, and as mentioned before, it is necessary to consider that the vulnerability period for any transmission is longer, as $T_{s}^{\prime}>T_{s}$ if $p_{e} \neq 0$.

In addition, since the relays also take part in the communication, the exposed area is enlarged when compared to a non-cooperative ARQ scheme. In this case, the ratio of additional transmissions that could be performed if the exposed terminal problem is avoided can be determined by

$\beta^{\prime}=\beta\left(1-p_{e}\right)+\beta_{\mathrm{C}-\mathrm{ARQ}} p_{e}$.

The value of $\beta$ is the same as the one computed with Eq. 11. For the computation of $\beta_{\mathrm{C}-\mathrm{ARQ}}$ it is necessary to consider the area exposed to the retransmissions by the relays, which is different from $E 2$. To proceed with this analysis we support our discussion with Fig. 3.

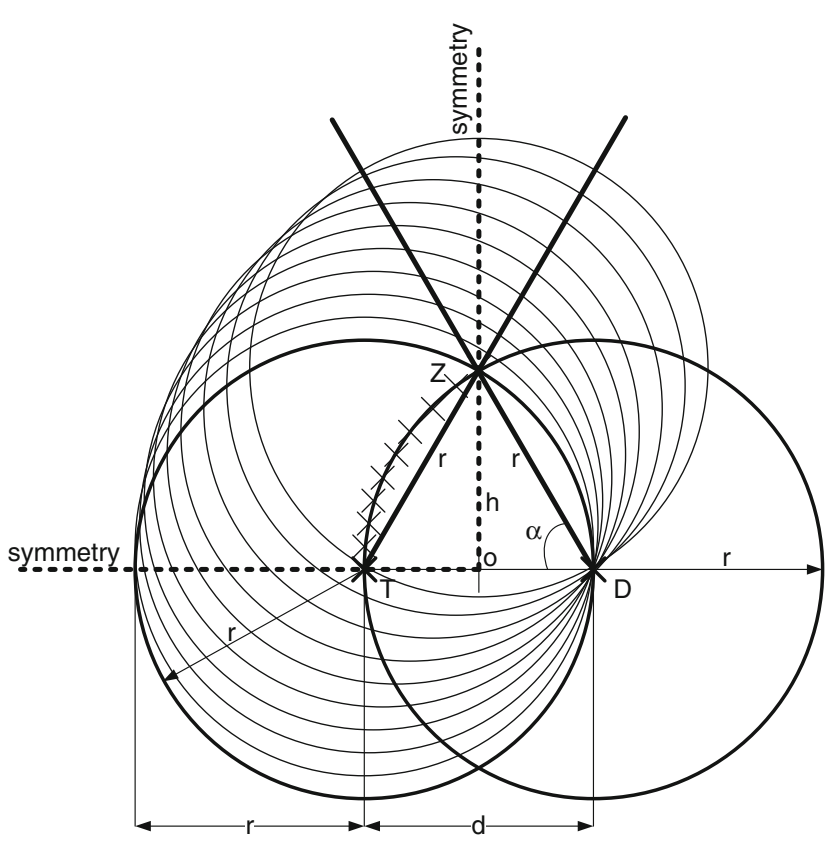

Fig. 3 Exposed area (description) 
In this figure we consider that station $\mathrm{T}$ is transmitting a packet to a destination $\mathrm{D}$, located at a distance $d$, which, by definition, is lower or equal than $r$ (transmission range of $\mathrm{T}$ ). The potential relays for this communication lie within the overlapping area of the transmitting and receiving ranges of both $\mathrm{T}$ and $\mathrm{D}$, respectively. Regarding the exposed terminal problem, the worst case will correspond to the one when the exposed area is maximized. This happens when the relays are placed at the edges of the overlapping area of the transmission ranges of $\mathrm{T}$ and $\mathrm{D}$. Therefore, if we want to compute the total area that can be affected by the exposed terminal problem, we should consider the area within the limits defined by the infinite circles of radius $r$ whose centers can be placed along the edge of the overlapping area of the transmission ranges of $\mathrm{T}$ and $\mathrm{D}$. As it is shown in the figure, in order to compute this area, it is possible to define two symmetry axes which define four regions with equal areas.

To make the exposition clearer, let have a look at Fig. 4. We can compute the exposed area as four times: (i) the area of the stripped sector of radius $(r+d)$ and angle $\alpha$, plus (ii) the area of the shadowed sector of radius $r$ and complementary angle of $\alpha$ (i. e., $\pi / 2-\alpha$ ), minus (iii) the area of the triangle delimited by the vertices DOZ. Accordingly, the area subject to the exposed terminal problem, denoted by $E_{\mathrm{C}-\mathrm{ARQ}}$, can be computed as

$$
E_{\mathrm{C}-\mathrm{ARQ}}=4\left[A_{s}(r+d, \alpha)+A_{s}\left(r, \frac{\pi}{2}-\alpha\right)-A_{t}\right] .
$$

$A_{s}(a, b)$ denotes the area of a sector of radius $a$ and angle $b$, and can be computed as $A(a, b)=(1 / 2) a^{2} b$. $A_{t}$ is the area of the triangle formed by the vertices DOZ, which can be computed as

$A_{t}=[(d / 2) r \sin (\alpha)] / 2$.

By simple observation of the figure it is possible to write that $\alpha=\arccos \left(\frac{d / 2}{r}\right)$ and, finally, the ratio of additional traffic that could be transmitted if the exposed terminal problem was solved can be expressed as

$\beta_{\mathrm{C}-\mathrm{ARQ}}=\left(\frac{B}{B+I}\right)(1-p)\left(\frac{E_{\mathrm{C}-\mathrm{ARQ}}}{E}\right)$.

With this value it is possible to compute the value of $\beta^{\prime}$ in Eq. 13 and thus evaluate the impact of the increased exposed area due to the use of a C-ARQ scheme at the MAC layer.

In the next section we present some numerical evaluation with a practical case study, also supported by computer simulations.

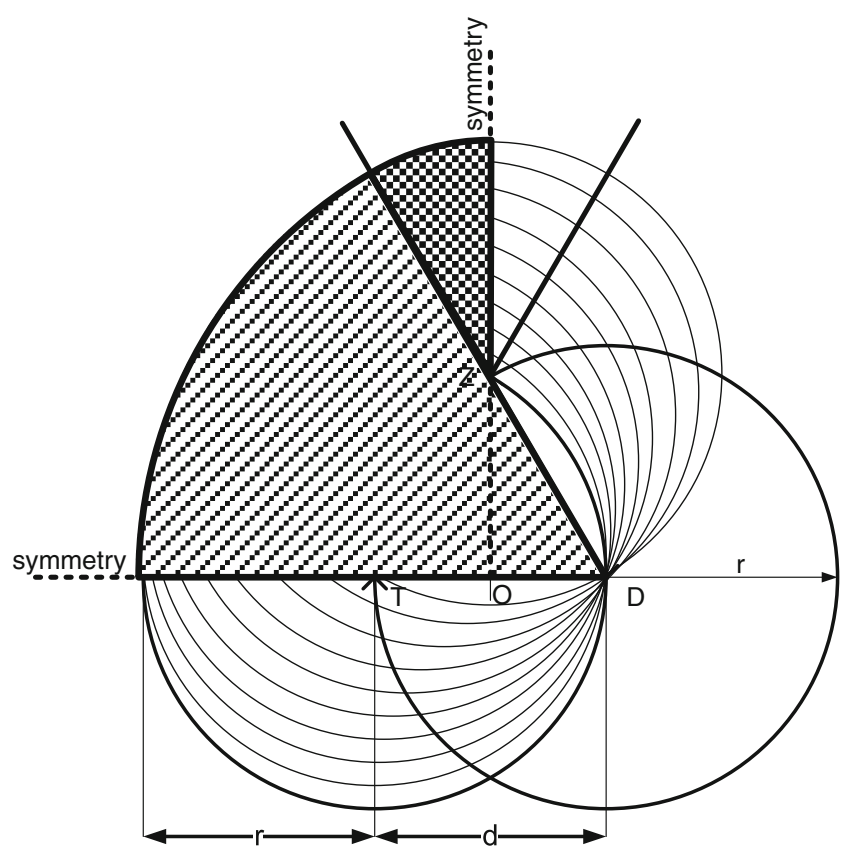

Fig. 4 Exposed area (calculation)

\section{Performance evaluation}

\subsection{Scenario}

In this section we evaluate the performance of two different ad hoc scenarios based on the IEEE 802.11 Standard MAC protocol:

1. A layout where a number of $M=5$ users are uniformly distributed in a given area, in LOS conditions. According to Eq. 9, a total of $4 M=20$ terminals are located in the surroundings of these users, also uniformly distributed. In this scenario we assume that the RTS/CTS handshake (as defined in the 802.11 Standard) is able to completely solve the hidden terminal problem so that we can focus on the analysis on the exposed terminal problem.

2. The same scenario as before, but considering that the exposed stations can know when they can transmit or not. By comparing this case with the previous one, it is possible to evaluate which the impact of the presence of exposed stations in a network is.

In both cases, we assume that all the users have the same traffic generation characteristics.

We have used the theoretical analysis presented in the previous section and we have also supported the results with simulations carried out with a $\mathrm{C}++$ simulator. The simulator executes the protocol rules without using any of the theoretical expressions presented in 
this paper. In all cases, the average packet error probability has been fixed to $p_{e}=0.5$ for the transmission of data and $p_{e}=0$ for control packets due to the use of the most robust coding and modulation scheme used for the control plane. The channel between the relays and the destination is assumed to be error-free as we assume that the active relays are very close to the destination. Although any other value could be used, we assume that exactly two successful retransmissions from the relays are required to attempt to decode the original packet at destination. Regarding the offered load to the network, we assume a homogeneous traffic distribution (all the stations contribute equally to the total offered load) and generate Poisson traffic with parameter $g$ and thus

$p=\frac{(g \tau)^{0}}{0 !} e^{-g \tau}=e^{-g \tau}$,

$p_{1}=\frac{(g \tau)^{1}}{1 !} e^{-g \tau}=g \tau e^{-g \tau}$,

$\beta^{\prime}=1-e^{g_{e} \tau}=>g_{e}=\frac{-\ln \left(1-\beta^{\prime}\right)}{\tau}$.

Recall, as defined in Section 3.2, that $\tau$ is the duration of the time slot and $p$ is the probability that no packet arrives in a given time slot. The length of data packets has an exponential distribution with average 1,500 bytes. According to [13], these are the size and distributions that better represent the data traffic of a WLAN. The rest of the parameters for both analysis and simulation are summarized in Table 1.

For each scenario, two ARQ schemes are compared:

1. Plain $A R Q$, if retransmissions are requested from the original source station. In this case, retransmission is scheduled a SIFS period after the detection of the failed transmission attempt from the source.

2. C-ARQ, if retransmissions are requested from a number of relays which overheard the original transmission. In this case, the cooperation phase is started one SIFS period after the detection of the failed transmission attempt from the source.

For the execution of the C-ARQ, we consider that the relays use the Persistent Relay CSMA (PRCSMA) protocol described in [5] (and further analyzed in [6]) to gain access to the channel. For completeness we review the operation of PRCSMA in the following subsection.

\subsection{PRCSMA overview}

PRCSMA is a protocol designed to coordinate the retransmission of the relays in a C-ARQ scheme. Whenever a destination receives a data packet with errors, it broadcasts a CFC packet. All the users which overheard the original transmission and receive this CFC packet become active relays and contend to get access to the channel in order to assist the destination. The operation of PRCSMA is essentially based on the rules of the IEEE 802.11 MAC protocol, except for the two following modifications:

1. The relays perform a backoff right after receiving the CFC broadcast by the destination asking for cooperation. This initial backoff is necessary to avoid a certain collision among all the relays willing to cooperate.

2. The relays do not expect any ACK for each retransmission as they are not the original source of the transmitted packet. Therefore, the overhead associated to the retransmissions can be reduced.

The closed-form equation to compute the value of $T_{\text {COOP }}$ in Eq. 12 within the context of PRCSMA can also be found in [5] and [6].

\subsection{Results}

The throughput, as defined in Eq. 1, is plotted in Fig. 5. The first observation is the good match between the model and the simulations.

It is worth noting that simulation results always show a slightly better throughput than those obtained with the theoretical analysis. The reason for this behavior is the assumption adopted in the theoretical analysis that considers the worst-case scenario in terms of exposed terminal area. In the analysis, we have considered that the destination is always located at the edge of the transmission range of the source, i.e., we have

Table 1 System parameters

\begin{tabular}{llll}
\hline Parameter & Value & Parameter & Value \\
\hline Data Tx. rate source & $6 \mathrm{Mbps}$ & Ctrl. Tx. rate source & $6 \mathrm{Mbps}$ \\
Data Tx. rate relays & $54 \mathrm{Mbps}$ & Ctrl. Tx. rate relays & $6 \mathrm{Mbps}$ \\
MAC header & 34 bytes & PHY preamble & $96 \mu s$ \\
DIFS & $50 \mu \mathrm{s}$ & SIFS & $10 \mu \mathrm{s}$ \\
ACK length & 14 bytes & SlotTime $(\tau)$ & $10 \mu s$ \\
RTS length & 20 bytes & CTS and CFC length & 14 bytes \\
Required retx. & 2 & Packet error prob. $\left(p_{e}\right)$ & 0.5 \\
\hline
\end{tabular}




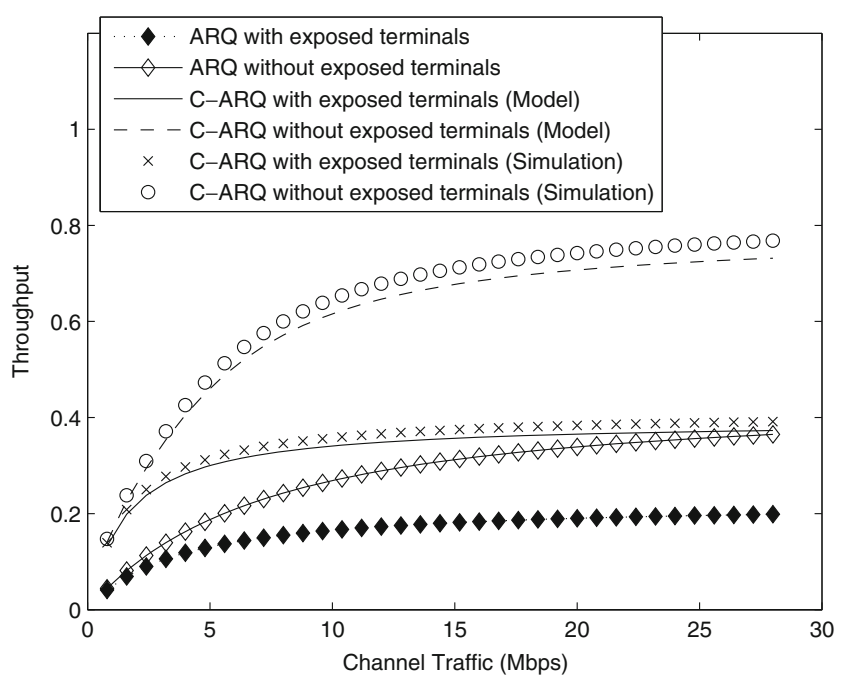

Fig. 5 Throughput

maximum distance between source and destination. In addition, the relays are always placed at the edges of the intersection of the transmission ranges of the source and the destination, thus maximizing the exposed area. Therefore, the analysis provides a lower-bound of the achievable throughput in the system in the presence of exposed terminals. However, when the simulation is executed, the distance between source and destination changes from packet to packet, as the traffic distribution is uniformly distributed among all the users in the scenario. In addition, the relays supporting a sourcedestination pair may not be always at the edge of the transmission range of both source and destination, thus reducing the exposed area as well. This reduction of the exposed area, opposed to the maximum exposed area assumed in the theoretical model, yields higher throughput in the network when the simulations are executed.

In any case, the results obtained through simulation show that the approximation in the theoretical model is fairly accurate, as the difference between model and simulation is always below $5 \%$.

In terms of the exposed terminal problem, it is possible to see that there is a remarkable difference between the performance of a network with and without exposed terminals. In fact, this difference becomes more outstanding in the cooperative case, where, as expected, the area exposed to the transmissions is larger.

In order to better evaluate this effect, we plot in Fig. 6 the ratio of exposed throughput to the total throughput, i.e., the ratio $U\left(g_{e}\right) / U$ (see Eq. 10). This ratio represents the percentage of traffic that cannot be conveyed in the network due to the exposed terminal

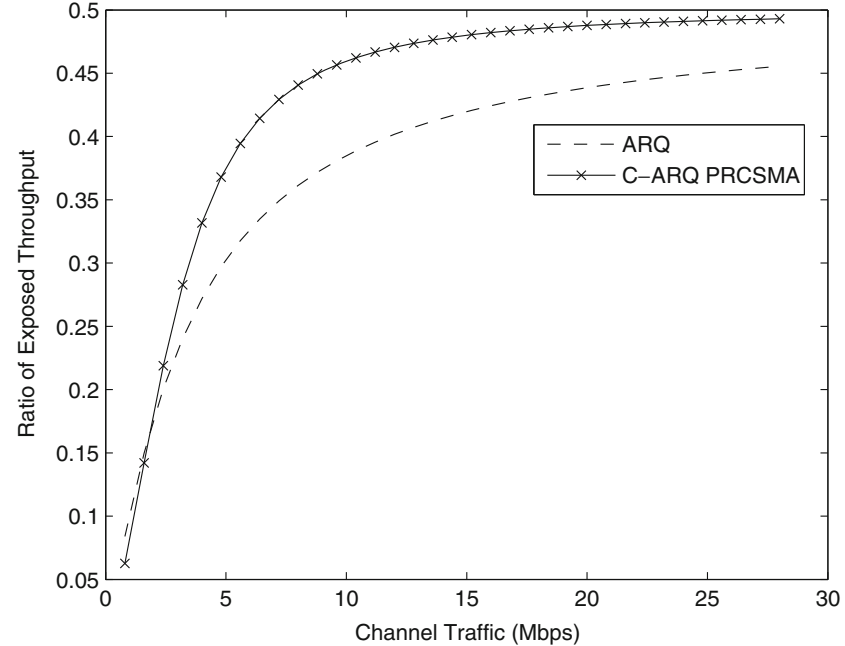

Fig. 6 Ratio of exposed throughput

problem. It is worth seeing that in the C-ARQ case, the fact that more than one station is involved in the link from the source to the destination enlarges the exposed area, as we already discussed in the previous section. However, it is also interesting to see that, as expected by Korakis et al. [8], although the exposed area is effectively enlarged, the total channel utilization is higher in the cooperative case. This means that, although the exposed area is larger, and thus more users may be exposed to a transmission, the duration of this exposition is shorter than in a non-cooperative ARQ due to the faster retransmissions, thus leading to an improved overall performance. Of course, this payoff depends directly on the effective transmission rate between the relays and destination, and thus it may exist situations where the obtained benefits in terms of channel utilization do not pay off the the enlarged exposed area.

In any case, it seems clear that the design of an efficient mechanism to combat the exposed terminal problem will benefit both schemes (with and without C-ARQ). Note that, as shown in Fig. 5, the performance of the network with C-ARQ could be improved in up to $100 \%$ if the exposed terminal problem could be solved. This will be our motivation for future work.

\section{Conclusions}

We have evaluated in this paper how a C-ARQ scheme executed at the MAC layer modifies the analysis of the hidden and exposed terminal problems in CSMA-based networks, such as for example, IEEE 802.11 networks. 
The hidden terminal problem remains almost unaltered except for the fact that the longer transmission times, due to retransmissions, are more vulnerable to potential hidden terminal transmissions. On the other hand, both numerical evaluation and computer simulations show that the C-ARQ scheme is more affected by the presence of exposed terminals than the non-cooperative ARQ scenario due to the enlargement of the exposed area of any transmission when a cooperative phase occurs. Therefore, a tradeoff should be carefully managed between the improved performance attained by the C-ARQ scheme and the exacerbation of the exposed terminal problem. Under some conditions, it may not be suitable to execute cooperation.

Future work will be aimed at extending this model to include the theoretical model of PRCSMA in the analysis and at designing efficient mechanisms to combat the exposed terminal problem in the C-ARQ scenario.

\section{References}

1. Nosratinia A, Hunter TE, Hedayat A (2004) Cooperative communications in wireless networks. IEEE Commun Mag 42(10):74-80

2. Dianati M, Ling X, Naik K, Shen X (2006) A nodecooperative ARQ scheme for wireless ad hoc networks. IEEE Trans Veh Technol 46:1032-1044
3. Zimmermann E, Herhold P, Fettweis F (2005) On the performance of cooperative relaying protocols in wireless networks. Eur Trans Telecomm 16:5-16

4. Cerruti I, Fumagalli A, Gupta P (2008) Delay model of singlerelay cooperative ARQ protocols in slotted radio network with poisson frame arrivals. IEEE/ACM Trans Netw 16: 371-382

5. Alonso-Zárate J, Kartsakli E, Verikoukis Ch, Alonso L (2008) Persistent RCSMA: a MAC protocol for a distributed cooperative ARQ scheme in wireless networks. EURASIP J Adv Sig Proc 2008:1-13. doi:10.1155/2008/817401

6. Alonso-Zárate J, Verikoukis C, Alonso L (2009) Performance analysis of a persistent relay carrier sensing multiple access protocol. IEEE Trans on Wireless Comms 8(12):58275831

7. Zhu H, Cao G (2006) rDCF: a relay enabled medium access control protocol for wireless ad hoc networks. IEEE Trans Mob Comput 5:1201-1214

8. Korakis T, Tao Z, Slutskiy Y, Panwar S (2007) A cooperative MAC protocol for ad hoc wireless networks. In: Proc. of the fifth annual IEEE international conference on pervasive computing and communications workshops

9. Wireless LAN medium access control (MAC) and physical layer (PHY) specifications. IEEE Standard 802.11 (2007)

10. Bianchi G (2000) Performance analysis of the IEEE 802.11 distributed coordination function. IEEE J Sel Areas Commun 18:535-547

11. Kleinrock L, Tobagi FA (1975) Packet switching in radio channels: part II - the hidden terminal problem in carrier sense multiple-access and the busy-tone solution. IEEE Trans Commun 23:1417-1433

12. Vassis D, Kormentzas G (2008) Performance analysis of IEEE 802.11 ad hoc networks in the presence of exposed terminals. Elsevier Ad hoc Networks 6:474-482

13. Yeo J, Youssef M, Agrawala A (2004) Characterizing the IEEE 802.11 traffic: the wireless side. University of Meryland, College Park, Tech. Rep. CS-TR-457 